\title{
Desigualdades sociodemográficas e causas de morte em menores de cinco anos no Estado de Pernambuco
}

\section{Sociodemographic inequalities and cause of \\ death among children aged under five years in the Brazilian State of Pernambuco}

Gabriela Ferraz Murakami 1 Maria José Bezerra Guimarães 2 Sílvia Wanick Sarinho 3
1,3 Universidade de Pernambuco. Av. Agamenon Magalhães, s.n. Santo Amaro. Recife, PE, Brasil. CEP: 50.100-010.

E-mail: gabimurakami@hotmail.com

2 Hospital Universitário Oswaldo Cruz. Recife, PE, Brasil.

\begin{abstract}
Objectives: to characterize mortality in children aged under five years in the three-year period, 2004-2006, identifying sociodemographic inequalities and differences in the cause of death among the sub-regions of the State of Pernambuco.

Methods: a cross-sectional study was carried out of 6721 deaths of children aged under five living in municipalities with death and alive newborn registers and these were divided up into four sub-regions. For the variables under study, inequalities in proportional mortality (chisquared) and mortality rates among children aged under five (MRU5) - ratio of rates and overmortality - were analyzed.

Results: among the sub-regions, the MRU5 varied from 18.3/1000NV (in the Metropolitan Region of Recife - RMR) to 28.4/1000NV (in the Sertão), the mean being 21.7/1000NV. The higher MRU5s for Mata, Agreste and Sertão compared to the MRU5 for the RMR represent an over-mortality rate of 1055. The proportional mortality differed $(p<0.05)$ as to place of death occurence, characteristics of municipality of residence (HDI, women's literacy, fecundity rate) specific basic causes of the chapters of perinatal diseases, infectious diseases and parasites. In the Sertão, the MRU5 that could be reduced to adequate care levels for pregnant women, delivery and newborns, by promoting health and adequate diagnosis and treatment were 1.6; 2.2 and 2.7 times higher than in the RMR.

Conclusions: mortality in children aged under five in Pernambuco revealed inequalities among the sub-regions.
\end{abstract}

Key words Child mortality, Causes of death, Health inequalities

\section{Resumo}

Objetivos: caracterizar a mortalidade em menores de cinco anos, no triênio 2004-2006, identificando desigualdades sociodemográficas e nas causas de morte entre as mesorregiões de Pernambuco.

Métodos: realizou-se um estudo transversal, com 6721 óbitos em menores de cinco anos residentes em municipios com Sistema de Informação sobre Mortalidade e Sistema de Informação sobre Nascidos Vivos consolidados, agregados em quatro mesorregiões. Para as variáveis estudadas, analisaram-se desigualdades na mortalidade proporcional (qui-quadrado) e nas taxas de mortalidade em menores de cinco anos (TMM5) - razão de taxas e sobremortalidade.

Resultados: entre as mesorregiões, a TMM5 variou de 18,3/1000NV (Região Metropolitana do Recife-RMR) a 28,4/1000NV (Sertão), com média de 21,7/1000NV. As maiores TMM5 da Mata, Agreste e Sertão em relação à TMM5 da RMR representaram uma sobremortalidade de 1055 óbitos. A mortalidade proporcional apresentou diferenças $(p<0,05)$ quanto ao local de ocorrência do óbito, características do município de residencia (IDH, analfabetismo feminino, taxa de fecundidade) e causas básicas específicas nos capitulos afecções perinatais, doenças infecciosas e parasitárias. No Sertão, as TMM5 reduziveis por adequada atenção à gestação-parto-RN, por ações de promoção à saúde e por diagnóstico e tratamento adequados foram 1,6; 2,2 e 2,7 vezes superiores às da RMR.

Conclusões: a mortalidade em menores de cinco anos em Pernambuco apresenta desigualdades entre as mesorregiões.

Palavras-chave Mortalidade na infância, Causas de óbito, Desigualdades em saúde 


\section{Introdução}

A taxa de mortalidade na infância, também denominada taxa de mortalidade em menores de cinco anos (TMM5), é mensurada pelo número de óbitos nesta faixa etária por mil nascidos vivos (NV). Em todas as regiões do mundo, a taxa de mortalidade na infância vem decrescendo. Entre 1990 e 2007, foi reduzida de 179 para $130 / 1000 \mathrm{NV}$ nos países com pior situação de desenvolvimento, de 103 para $74 / 1000 \mathrm{NV}$ nos que apresentam situação intermediária de desenvolvimento e de 10 para $6 / 1000$ NV nos considerados industrializados. ${ }^{1}$

No Brasil, a TMM5 apresentou declínio de $46,6 \%$, entre $1990 \quad(53,7 / 1000 \mathrm{NV})$ e 2005 $(28,7 / 1000 \mathrm{NV}), 2$ aproximando-se da meta estabelecida pela Declaração do Milênio. O Brasil, como signatário desta declaração, tem o compromisso de reduzir a mortalidade na infância em dois terços, entre 1990 e 2015, devendo apresentar uma TMM5 de 17,9/1000 $\mathrm{NV}^{2}$ no final desse período. O Nordeste, apesar de ser a região do país onde vem ocorrendo o maior declínio, ainda é a que apresenta a maior TMM5 (38,9/1000 NV, em 2005), com valores cerca de duas vezes superiores aos da Região Sul (18,0/1000 NV). ${ }^{2}$ Além de alcançar a meta do milênio, o Brasil tem o desafio de reduzir as desigualdades internas, diminuindo a importante heterogeneidade da TMM5 entre regiões, estados e municípios. O foco na redução das desigualdades parte do pressuposto de que todos os indivíduos de uma sociedade devem ter justa oportunidade para desenvolver seu pleno potencial e que as diferenças que conformam desvantagens podem ser evitadas, pois configuram iniquidades. 3

A redução da mortalidade em menores de cinco anos depende de intervenções sobre os seus determinantes. A cadeia causal da mortalidade na infância é complexa, com a participação de fatores de várias dimensões e que podem ser hierarquizados de acordo com sua precedência no tempo e relevância para o desfecho. Os determinantes considerados em um nível distal, como os fatores socioeconômicos, influenciam os que se situam no nível intermediário, referentes ao ambiente e à atenção à saúde, que, por sua vez, influenciam os determinantes proximais, considerados os que agem mais diretamente sobre a mortalidade na infância.4,5 Dentre esses últimos, destacam-se o peso ao nascer e a idade gestacional. 5,6

No Brasil, a maior parcela dos óbitos na infância ocorre antes da criança completar o primeiro ano de vida, grupo etário em que predominam as afecções perinatais como causa básica de morte. Na faixa etária entre um e quatro anos, destacam-se as diarréias, infecções respiratórias, desnutrição e acidentes entre as causas básicas de óbito.

Muitas causas básicas de morte na infância são consideradas total ou parcialmente evitáveis pela ação efetiva dos serviços de saúde. ${ }^{7}$ Dessa forma, ainda que se reconheça a necessidade de mudanças estruturais para redução das iniquidades relacionadas ao risco de adoecimento e morte em menores de cinco anos, é fundamental a discussão sobre como as ações e serviços de saúde se distribuem reproduzindo historicamente desigualdades socioeconômicas. ${ }^{8}$

Diante das importantes disparidades brasileiras no risco de morte das crianças e do papel que o setor saúde pode exercer para minimizá-las, este estudo tem como objetivo caracterizar a mortalidade na infância em Pernambuco, no triênio 2004-2006, identificando e mensurando desigualdades sociodemográficas e nas causas de morte em menores de cinco anos entre as mesorregiões do Estado.

\section{Métodos}

Os dados estudados referem-se ao Estado de Pernambuco, que está localizado no centro Leste da região Nordeste do Brasil, com área de 98.937,8 $\mathrm{km}^{2}, 8.810 .318$ habitantes (2009) e densidade demográfica de $89,05 \mathrm{hab} / \mathrm{km}^{2}$. Cerca de $75 \%$ de sua população vivem em área urbana. 9 Pernambuco possui 184 municípios e um distrito estadual, agrupados em cinco mesorregiões: Região Metropolitana do Recife (RMR), Mata, Agreste, Sertão e Sertão do São Francisco. Para fins deste estudo, as duas últimas mesorregiões foram agregadas, resultando na mesorregião denominada Sertão.

Realizou-se um estudo tipo corte transversal, com a inclusão dos óbitos em menores de cinco anos e dos nascidos vivos residentes em Pernambuco, ocorridos no triênio 2004-2006 e captados pelos sistemas de informação sobre mortalidade (SIM) e nascidos vivos (Sinasc). Como ainda persistem diferenciais na cobertura e qualidade dos dados provenientes desses sistemas, os municípios do Estado com informações consideradas adequadas foram selecionados e, posteriormente, agrupados em mesorregiões. Para tal seleção, foram utilizados os critérios propostos por Szwarcwald et al. ${ }^{10}$ para o cálculo direto de taxas de mortalidade, quanto à adequação dos dados provenientes do SIM e do Sinasc. Desse modo, foram calculados todos os indicadores e aplicados os pontos de corte propostos pelos referidos autores, para os 184 municípios do Estado: a) taxa geral de mortalidade padronizada por 
idade maior do que 6,75 por 1000 habitantes. A padronização foi realizada pelo método indireto, utilizando-se como referência a população de Pernambuco do ano 2000; b) taxa de natalidade maior do que 16 por 1000 habitantes; c) proporção de óbitos por causas mal definidas menor do que $20 \%$; d) desvio médio da taxa geral de mortalidade padronizada por idade menor do que $10 \%$; e) desvio médio da taxa de natalidade menor do que $10 \%$. De acordo com esses critérios, foram selecionados 99 municípios das quatro mesorregiões consideradas: RMR (14/14), Mata (32/43), Agreste (25/71) e Sertão (28/56).

Ao todo, foram estudados 6721 óbitos em menores de cinco anos e 309.544 nascidos vivos residentes nos municípios selecionados. Como variáveis sociodemográficas selecionaram-se idade, sexo, local de ocorrência do óbito e características do município de residência. As variáveis raça/cor e grau de instrução da mãe não foram selecionadas devido ao alto percentual de dados ignorados no banco do SIM. A idade foi categorizada de acordo com os dois principais grupos etários da mortalidade na infância: menores de um ano e um a quatro anos.

Para caracterizar o município de residência foram utilizadas três variáveis referentes ao ano 2000: índice de desenvolvimento humano (IDH), taxa de analfabetismo feminino em maiores de 15 anos e taxa de fecundidade total. 9 Para essas variáveis, os óbitos foram estratificados em tercis. Porém, as mortes em residentes nos municípios da RMR não foram considerados na determinação dos estratos, pois caso fossem incluídos, somente essa mesorregião apresentaria óbito no melhor tercil.

Quanto às causas básicas, para os dois principais grupos etários, os óbitos foram descritos por capítulo e causas específicas da Classificação Estatística Internacional de Doenças e Problemas Relacionados à Saúde - Décima Revisão (CID-10), ${ }^{11}$ além de causas evitáveis. Adotou-se a classificação de evitabilidade da causa básica proposta por Malta et al., 12 que as agrupam em seis categorias: a) reduzíveis por imunoprevenção; b) reduzíveis por adequada atenção à mulher na gestação e parto, e ao recém nascido; c) reduzíveis por ações adequadas de diagnóstico e tratamento; d) reduzíveis por ações adequadas de promoção à saúde, vinculadas a ações adequadas de atenção à saúde; e) causas de morte mal definidas; f) demais causas.

Para descrição dos óbitos em menores de cinco anos por mesorregião de residência, calculou-se a mortalidade proporcional (\%) para todas as variáveis estudadas. Também, para todas as variáveis, com exceção da causa básica por capítulo e causa especí- fica, obteve-se a TMM5 para o triênio estudado (por mil nascidos vivos). Para o cálculo da TMM5 por faixa etária, utilizou-se o número de nascidos vivos como denominador dos dois grupos considerados (menores de um ano e um a quatro anos). Os óbitos com dados ignorados foram excluídos para efeito do cálculo da mortalidade proporcional e TMM5, fato que ocorreu apenas para as variáveis sexo e local de ocorrência do óbito ( $0,3 \%$ na Mata e Agreste, a $0,6 \%$ na RMR). As diferenças na mortalidade proporcional entre as mesorregiões foram testadas por meio do teste qui-quadrado $(\alpha=0,05)$ e, quando necessário, do teste exato de Fisher $(\alpha=0,05)$. Para a variável causa básica, por faixa etária, esses testes estatísticos foram aplicados para identificação de diferenças entre os capítulos e entre as causas específicas de cada capítulo por mesorregião.

Foram obtidas duas medidas de desigualdade da mortalidade em menores de cinco anos entre as mesorregiões, tendo-se como referência a RMR: a razão de taxas e a sobremortalidade. A razão de taxas foi obtida dividindo-se a TMM5 de cada mesorregião pela TMM5 da RMR, com intervalo de confiança de $95 \% .13$

Para a obtenção da sobremortalidade (excesso de óbitos) em menores de cinco anos, inicialmente calculou-se o número de óbitos esperados nessa faixa etária na Mata, Agreste e Sertão, supondo que as TMM5 dessas mesorregiões fossem iguais a da RMR (taxa de referência). Para isso, a TMM5 da RMR foi multiplicada pelo número de nascidos vivos de cada uma das outras mesorregiões, chegando-se, após divisão pelo fator de multiplicação da taxa (1000), ao número de óbitos esperados em cada mesorregião. A diferença entre os óbitos observados e esperados correspondeu à sobremortalidade absoluta, ou seja, ao número absoluto de óbitos em excesso. Dividindo-se a sobremortalidade absoluta pelo número de óbitos observados e multiplicando-se por 100 , chegou-se à sobremortalidade relativa, ou seja, ao excesso relativo de óbitos em menores de cinco anos de cada mesorregião em relação à mesorregião de referência.

O estudo foi aprovado pelo Comitê de Ética em Pesquisa da Universidade de Pernambuco, sob o número 150/08.

\section{Resultados}

No período estudado, ocorreram 6721 óbitos em menores de cinco anos residentes nos municípios selecionados, correspondendo a uma taxa de mortalidade de 21,7/1000NV. Entre as mesorregiões, a maior TMM5 foi observada no Sertão $(28,4 / 1000$ 
NV) e a menor na RMR (18,3/1000 NV), correspondendo a uma razão de taxas de 1,55 (IC95\%: 1,45 1,65). Nas mesorregiões da Mata e do Agreste, a TMM5 (24,2 e 24,9/1000NV, respectivamente) mostrou-se mais próxima da observada no Sertão do que da TMM5 da RMR (Tabela 1).

Em relação à faixa etária (Tabela 1), ocorreram seis óbitos em menores de um ano para cada óbito entre um e quatro anos em todas as mesorregiões, à exceção do Sertão, na qual a relação foi mais elevada $(7: 1)$. Entre as mesorregiões, a mortalidade proporcional por faixa etária não apresentou diferenças estatisticamente significantes. Nos menores de um ano, a taxa de mortalidade variou entre 15,9/1000 NV (RMR) e 24,8/1000 NV (Sertão), com média de $18,7 / 1000 \mathrm{NV}$. A taxa nessa faixa etária apresentou o mesmo comportamento da TMM5: a RMR com melhor situação e o Sertão com a pior. A mortalidade em menores de um ano, no Sertão, foi 1,57 vezes superior ao da RMR, conforme foi observado pela razão de taxas (IC95\%: 1,46 - 1,68); no Agreste, 1,34 vezes (IC95\%: $1,25-1,44$ ) e na Mata, 1,30 vezes (IC95\%: 1,21 - 1,40).

Para o grupo etário de um a quatro anos (Tabela 1), a taxa de mortalidade situou-se entre 2,5/1000 NV (RMR) e 3,6/1000 NV (Mata e Agreste), com média de 3,0/1000 NV. A taxa no Sertão (3,5/1000 $\mathrm{NV}$ ) foi discretamente inferior à observada na Mata e no Agreste. Essas duas últimas mesorregiões apresentaram taxas de mortalidade na faixa etária de um a quatro anos entre $43 \%$ e $46 \%$ superior ao observado na RMR.

Se todas as mesorregiões apresentassem a mesma TMM5 da RMR, seriam esperados 5666 óbitos em menores de cinco anos residentes nos municípios selecionados (Tabela 1). Portanto, deixariam de ocorrer 1055 óbitos (excesso absoluto de óbitos) ou 18,6\% do total de mortes observadas no Estado (excesso relativo de óbitos). A maior sobremortalidade foi verificada no Sertão: 447 óbitos ou $55,2 \%$ do total de mortes observadas na mesorregião. No Agreste e na Mata, esse excesso correspondeu a $336(36,4 \%)$ e 273 óbitos $(32,6 \%)$, respectivamente.

Em relação às variáveis sociodemográficas (Tabela 2), apenas a mortalidade proporcional por sexo não apresentou diferença estatística entre as mesorregiões. Apesar de a maioria dos óbitos ter ocorrido em estabelecimentos de saúde, em todas as mesorregiões, identificaram-se diferenças na mortalidade proporcional por essa variável entre as mesorregiões de Pernambuco $(p<0,001)$. Na RMR, 95,7\% dos óbitos ocorreram em ambiente hospitalar e 3,5\%, em domicílio. Nas demais mesorregiões, a proporção de óbitos domiciliares variou entre 13,9\% (Sertão) e $17,6 \%$ (Mata), quatro a seis vezes maior que a observada na RMR. Em relação ao IDH, analfabetismo feminino e taxa de fecundidade, mais de $95 \%$ dos óbitos ocorreram em residentes em municípios pertencentes ao estrato com melhor condição da RMR e, nas demais mesorregiões, a mortalidade proporcional apresentou menor heterogeneidade entre os três estratos dessas variáveis.

Em todas as mesorregiões, a TMM5 foi maior no sexo masculino e nos estratos de municípios com pior situação do IDH, do analfabetismo feminino e da taxa de fecundidade, com exceção dessa última variável para a RMR, na qual a maior TMM5 foi observada no estrato com situação intermediária (Tabela 3). A razão de taxas evidenciou desigualdades entre as mesorregiões em quase todas as categorias das variáveis sociodemográficas $(p<0,05)$. Comparando-se as mesorregiões do interior do Estado com a RMR por meio das razões de taxas, destacam-se: a) TMM5 em domicílio do Sertão, Agreste e Mata 6,20; 6,85 e 6,77 vezes superior, respectivamente à da RMR; b) no Sertão, TMM5 $50 \%$ e $63 \%$ maior que a da RMR nos sexos masculino e feminino, respectivamente; c) nos estratos de municípios com pior IDH do Sertão, TMM5 61\% superior a da RMR; c) no estrato de municípios do Sertão com menor proporção de analfabetismo, TMM5 $62 \%$ superior a da RMR; d) no estrato de municípios com taxa de fecundidade mais elevada, TMM5 77\%, 70\% e 45\% mais elevada no Sertão, Agreste e Mata, respectivamente, em relação à taxa da RMR. Por outro lado, ressalta-se que no grupo de municípios com mais de $33,1 \%$ de analfabetismo feminino, não foram observadas diferenças significantes entre as TMM5 do Sertão, Agreste e Mata quando comparadas com a TMM5 da RMR.

Em menores de um ano (Tabela 4), foram identificadas diferenças entre as mesorregiões relacionadas à distribuição proporcional das causas básicas por capítulo da CID-10 $(p<0,001)$. Em todas, houve predominância das afecções perinatais, com discreta superioridade da proporção observada no Sertão e na RMR em relação às demais. Na RMR e no Agreste, as malformações congênitas apareceram em segundo lugar na distribuição das causas, seguidas das doenças infecciosas e parasitárias. $\mathrm{Na}$ Mata e no Sertão, as doenças infecciosas e parasitárias ocuparam o segundo lugar seguidas das malformações congênitas. Em todas as mesorregiões, as doenças do aparelho respiratório surgiram em quarto lugar com mortalidade proporcional entre 4,6 e $8,9 \%$. A proporção de causas mal definidas na Mata, Agreste e Sertão situou-se entre 


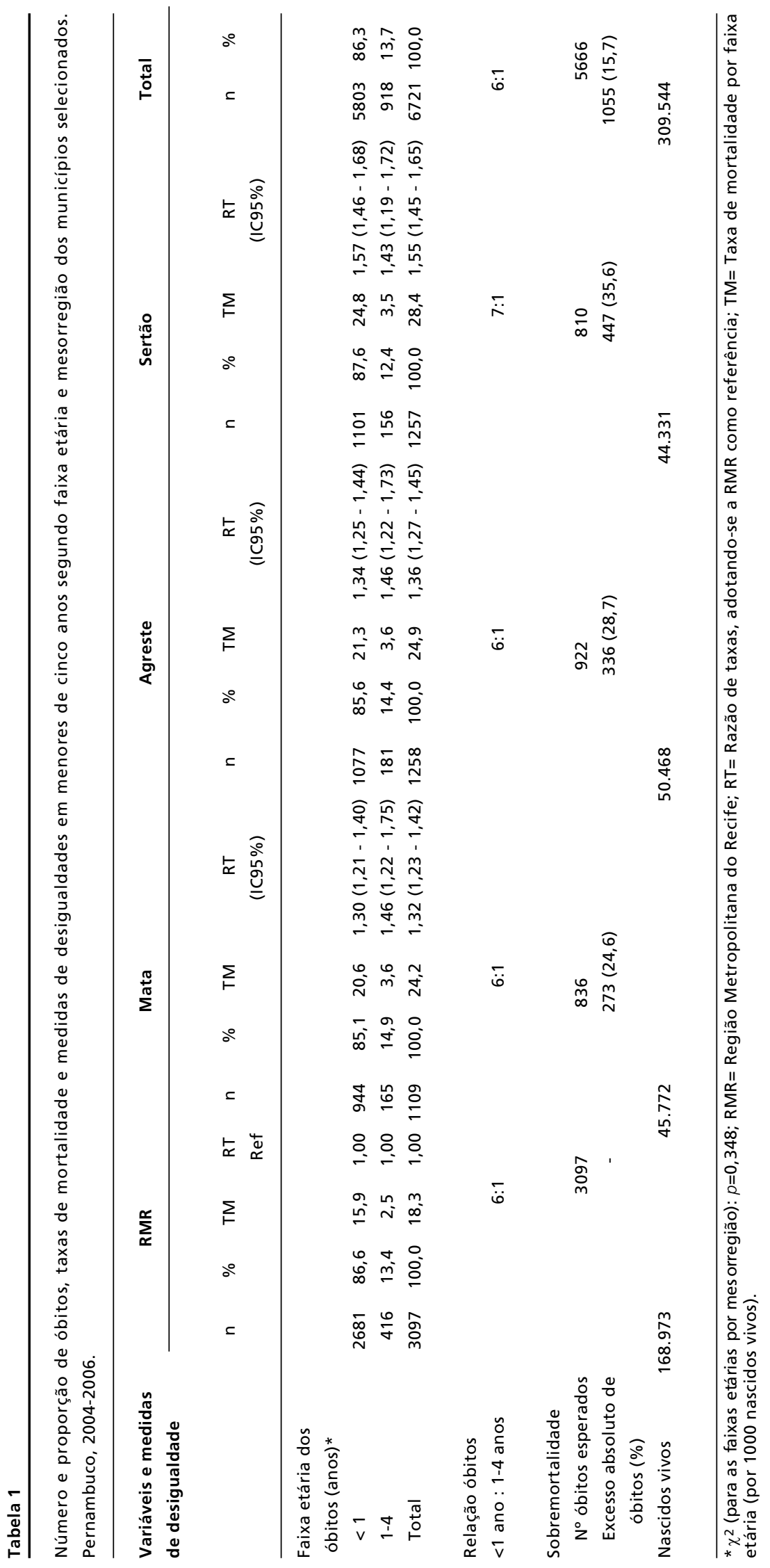




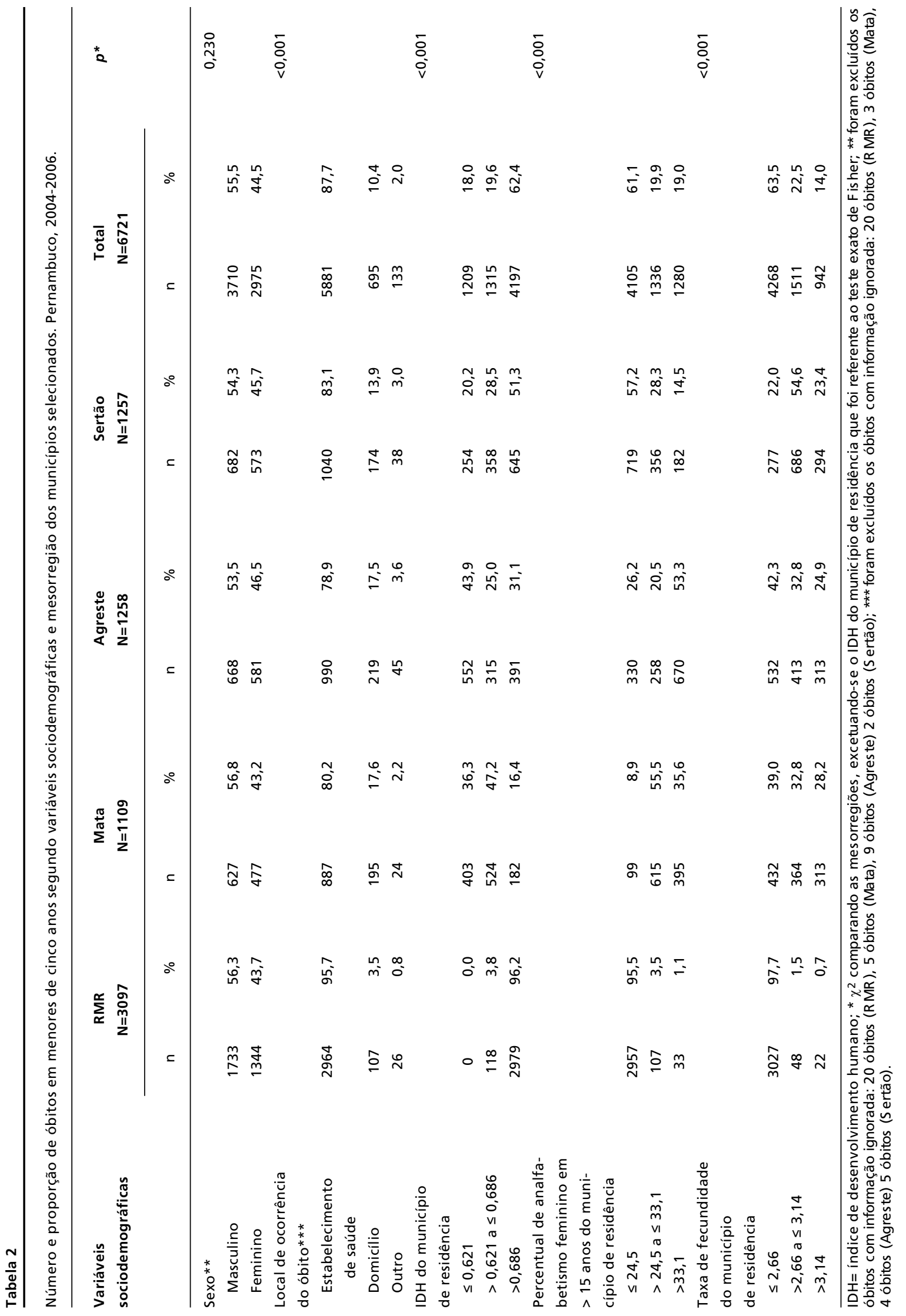




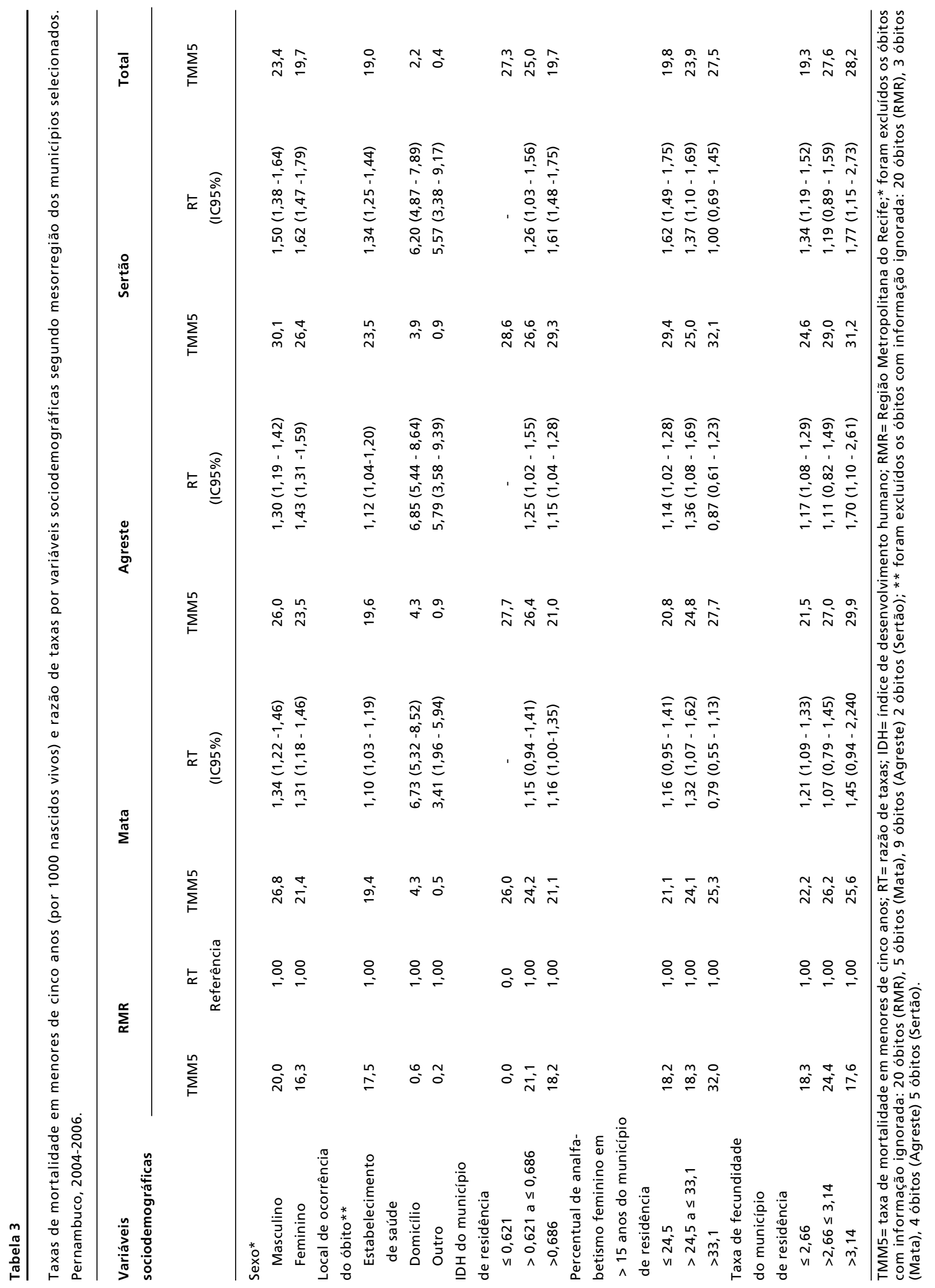


Número e percentual dos óbitos em menores de cinco anos por causa básica segundo faixa etária e mesorregião dos municípios selecionados. Pernambuco, 2004-2006.

\begin{tabular}{|c|c|c|c|c|c|c|c|c|c|c|c|}
\hline \multirow{2}{*}{$\begin{array}{l}\text { Capítulo (CID-10)* } \\
\text { Causa específica }\end{array}$} & \multicolumn{2}{|c|}{ RMR } & \multicolumn{2}{|c|}{ Mata } & \multicolumn{2}{|c|}{ Agreste } & \multicolumn{2}{|c|}{ Sertão } & \multicolumn{2}{|c|}{ Total } & \multirow[t]{2}{*}{$p^{* * *}$} \\
\hline & $\mathrm{n}$ & $\%$ & $\mathrm{n}$ & $\%$ & $\mathrm{n}$ & $\%$ & $\mathrm{n}$ & $\%$ & $\mathrm{n}$ & $\%$ & \\
\hline \multicolumn{12}{|l|}{ Menores de um ano* } \\
\hline Afecções perinatais (P00-P96) & 1550 & 58,0 & 490 & 51,9 & 534 & 49,6 & 663 & 60,3 & 3237 & 55,9 & $<0,001$ \\
\hline Afecções maternas (P00) & 370 & 13,8 & 38 & 4,0 & 43 & 4,0 & 27 & 2,5 & 478 & 8,2 & \\
\hline Complicações da gravidez (P01) & 236 & 8,8 & 39 & 4,1 & 32 & 3,0 & 24 & 2,2 & 331 & 5,7 & \\
\hline $\begin{array}{l}\text { Complicações da placenta, } \\
\text { cordão e membrana (P02) }\end{array}$ & 196 & 7,3 & 39 & 4,1 & 28 & 2,6 & 31 & 2,8 & 294 & 5,1 & \\
\hline $\begin{array}{l}\text { Baixo peso e prematuridade } \\
\text { (P05-P07) }\end{array}$ & 57 & 2,1 & 29 & 3,1 & 63 & 5,8 & 77 & 7,0 & 226 & 3,9 & \\
\hline Hipoxia perinatal (P20-P21) & 81 & 3,0 & 36 & 3,8 & 51 & 4,7 & 72 & 6,5 & 240 & 4,1 & \\
\hline $\begin{array}{l}\text { Transtornos respiratórios } \\
\text { do RN (P22-P28) }\end{array}$ & 354 & 13,2 & 178 & 18,9 & 181 & 16,8 & 235 & 21,4 & 948 & 16,4 & \\
\hline $\begin{array}{l}\text { Infecção/septicemia perinatal } \\
\text { (P36 e P39) }\end{array}$ & 134 & 5,0 & 60 & 6,4 & 76 & 7,1 & 114 & 10,4 & 384 & 6,6 & \\
\hline Outras afecções perinatais & 122 & 4,6 & 71 & 7,5 & 60 & 5,6 & 83 & 7,5 & 336 & 5,8 & \\
\hline Malformação congênita (Q00-Q99) & 555 & 20,8 & 119 & 12,6 & 152 & 14,1 & 107 & 9,7 & 933 & 16,1 & 0,519 \\
\hline $\begin{array}{l}\text { Do sistema nervoso central } \\
\text { (Q00-Q07) }\end{array}$ & 94 & 3,5 & 24 & 2,5 & 26 & 2,4 & 22 & 2,0 & 166 & 2,9 & \\
\hline $\begin{array}{l}\text { Do aparelho circulatório } \\
\text { (Q20-Q28) }\end{array}$ & 200 & 7,5 & 45 & 4,8 & 45 & 4,2 & 33 & 3,0 & 323 & 5,6 & \\
\hline Outras malformações & 261 & 9,8 & 50 & 5,3 & 81 & 7,5 & 52 & 4,7 & 444 & 7,7 & \\
\hline $\begin{array}{l}\text { Doenças infecciosas e } \\
\text { parasitárias (A00-B99) }\end{array}$ & 191 & 7,1 & 120 & 12,7 & 119 & 11,0 & 128 & 11,6 & 558 & 9,6 & $<0,001$ \\
\hline $\begin{array}{l}\text { Doenças infecciosas } \\
\text { intestinais (A02-A09) }\end{array}$ & 142 & 5,3 & 110 & 11,7 & 106 & 9,8 & 107 & 9,7 & 465 & 8,0 & \\
\hline Septicemia (A40-A41) & 10 & 0,4 & 4 & 0,4 & 7 & 0,6 & 16 & 1,5 & 37 & 0,6 & \\
\hline Outras & 39 & 1,5 & 6 & 0,6 & 6 & 0,6 & 5 & 0,5 & 56 & 1,0 & \\
\hline $\begin{array}{l}\text { Doenças do aparelho } \\
\text { respiratório (J00-J99) }\end{array}$ & 156 & 5,8 & 68 & 7,2 & 96 & 8,9 & 51 & 4,6 & 371 & 6,4 & 0,662 \\
\hline Pneumonia (J12-J18) & 118 & 4,4 & 46 & 4,9 & 69 & 6,4 & 37 & 3,4 & 270 & 4,7 & \\
\hline Outras & 38 & 1,4 & 22 & 2,3 & 27 & 2,5 & 14 & 1,3 & 101 & 1,7 & \\
\hline $\begin{array}{l}\text { Doenças endócrinas e } \\
\text { nutricionais (E00-E90) }\end{array}$ & 48 & 1,8 & 47 & 5,0 & 53 & 4,9 & 40 & 3,6 & 188 & 3,2 & 0,673 \\
\hline Desnutrição (E41-E46) & 43 & 1,6 & 41 & 4,3 & 50 & 4,6 & 36 & 3,3 & 170 & 2,9 & \\
\hline Outras & 5 & 0,2 & 6 & 0,6 & 3 & 0,3 & 4 & 0,4 & 18 & 0,3 & \\
\hline Causas mal definidas (R00-R99) & 13 & 0,5 & 50 & 5,3 & 67 & 6,2 & 59 & 5,4 & 189 & 3,3 & \\
\hline Outros capítulos & 160 & 6,0 & 50 & 5,3 & 56 & 5,2 & 52 & 4,7 & 318 & 5,5 & \\
\hline Total & 2673 & 100,0 & 944 & 100,0 & 1077 & 100,0 & 1100 & 100,0 & 5794 & 100,0 & \\
\hline
\end{tabular}


Número e percentual dos óbitos em menores de cinco anos por causa básica segundo faixa etária e mesorregião dos municípios selecionados. Pernambuco, 2004-2006.

\begin{tabular}{|c|c|c|c|c|c|c|c|c|c|c|c|}
\hline \multirow{2}{*}{$\begin{array}{l}\text { Capítulo (CID-10)* } \\
\text { Causa específica }\end{array}$} & \multicolumn{2}{|c|}{ RMR } & \multicolumn{2}{|c|}{ Mata } & \multicolumn{2}{|c|}{ Agreste } & \multicolumn{2}{|c|}{ Sertão } & \multicolumn{2}{|c|}{ Total } & \multirow[t]{2}{*}{$p * * *$} \\
\hline & $\mathrm{n}$ & $\%$ & $\mathrm{n}$ & $\%$ & $\mathrm{n}$ & $\%$ & $\mathrm{n}$ & $\%$ & $\mathrm{n}$ & $\%$ & \\
\hline \multicolumn{12}{|l|}{1 a 4 anos** } \\
\hline $\begin{array}{l}\text { Doenças do aparelho } \\
\text { respiratório (J00-J99) }\end{array}$ & 89 & 21,0 & 32 & 19,4 & 33 & 18,2 & 33 & 21,0 & 187 & 20,2 & 0,005 \\
\hline Pneumonia (J15-J18) & 65 & 15,3 & 15 & 9,1 & 25 & 13,8 & 16 & 10,2 & 121 & 13,1 & \\
\hline Outras & 24 & 5,7 & 17 & 10,3 & 8 & 4,4 & 17 & 10,8 & 66 & 7,1 & \\
\hline Causas externas (V01-Y98) & 72 & 17,0 & 31 & 18,8 & 43 & 23,8 & 31 & 19,7 & 177 & 19,1 & 0,276 \\
\hline $\begin{array}{l}\text { Acidentes não relacionados a } \\
\text { transporte (W06-X59) }\end{array}$ & 37 & 8,7 & 18 & 10,9 & 27 & 14,9 & 17 & 10,8 & 99 & 10,7 & \\
\hline Acidentes de transporte (V02-V8 & 9) 12 & 2,8 & 5 & 3,0 & 7 & 3,9 & 10 & 6,4 & 34 & 3,7 & \\
\hline Outras & 23 & 5,4 & 8 & 4,8 & 9 & 5,0 & 4 & 2,5 & 44 & 4,7 & \\
\hline $\begin{array}{l}\text { Doenças infecciosas e } \\
\text { parasitárias (A00-B99) }\end{array}$ & 59 & 13,9 & 25 & 15,2 & 28 & 15,5 & 27 & 17,2 & 139 & 15,0 & 0,738 \\
\hline $\begin{array}{l}\text { Doenças infecciosas } \\
\text { intestinais (A01-A09) }\end{array}$ & 36 & 8,5 & 14 & 8,5 & 16 & 8,8 & 13 & 8,3 & 79 & 8,5 & \\
\hline Outras & 23 & 5,4 & 11 & 6,7 & 12 & 6,6 & 14 & 8,9 & 60 & 6,5 & \\
\hline Causas mal definidas (R00-R99) & 10 & 2,4 & 17 & 10,3 & 10 & 5,5 & 7 & 4,5 & 44 & 4,7 & \\
\hline Outros capítulos & 194 & 45,8 & 60 & 36,4 & 67 & 37,0 & 59 & 37,6 & 380 & 41,0 & \\
\hline Total & 424 & 100,0 & 165 & 100,0 & 181 & 100,0 & 157 & 100,0 & 927 & 100,0 & \\
\hline
\end{tabular}

* $\chi^{2}$ relacionado aos Capítulos da CID-10 por mesorregião na faixa etária de menores de um ano: $p<0,001 ; * * \chi^{2}$ relacionado aos Capítulos da CID-10 por mesorregião na faixa etária de $1-4$ anos: $p=0,015 ; * * * \chi^{2}$ relacionado às caus as específicas de cada Capítulo da CID-10 por mes orregião, excetuando-se às causas específicas das doenças infecciosas e parasitárias e das doenças endócrinas e nutricionais, em menores de um ano, e das causas externas, na faixa de 1-4 anos, em que se utilizou o tes te exato de Fisher.

5,3 e $6,2 \%, 10$ a 12 vezes superiores à verificada na $\operatorname{RMR}(0,5 \%)$.

Em relação às causas específicas (Tabela 4), em menores de um ano, foram observadas diferenças, entre as mesorregiões, na mortalidade proporcional nos capítulos referentes às afecções perinatais e às doenças infecciosas e parasitárias $(p<0,001)$. No banco de dados do SIM, consta que $29,9 \%$ dos óbitos em menores de um ano, em residentes na RMR, foram decorrentes de três causas pertencentes ao capítulo das afecções perinatais: afecções maternas, complicações da gravidez e complicações da placenta, cordão e membranas. Na Mata, Agreste e Sertão, essa proporção foi de $12,1 \%, 9,6 \%$ e 7,5\%, respectivamente. Ainda em menores de um ano, nessas mesorregiões, os transtornos respiratórios do recém-nascido, a infecção/septicemia perinatal, o baixo peso/prematuridade e a hipóxia perinatal apre- sentaram mortalidade proporcional superior à observada na RMR. A proporção de doenças infecciosas intestinais (causa específica componente do capítulo das doenças infecciosas e parasitárias) na Mata, Agreste e Sertão foi praticamente o dobro da identificada na RMR.

Entre as mesorregiões, no grupo etário de um a quatro anos (Tabela 4), foram observadas diferenças na mortalidade proporcional por capítulo $(p=0,015)$. À exceção do Agreste, as doenças do aparelho respiratório predominaram como causas básicas de morte, em todas as mesorregiões, seguidas das causas externas, doenças infecciosas e parasitárias e causas mal definidas. No Agreste, as causas externas superaram as doenças do aparelho respiratório. Na Mata, mais de $10 \%$ dos óbitos de um a quatro anos não tiveram causa básica definida, o dobro do percentual encontrado no Agreste e no Sertão e o quádruplo do 


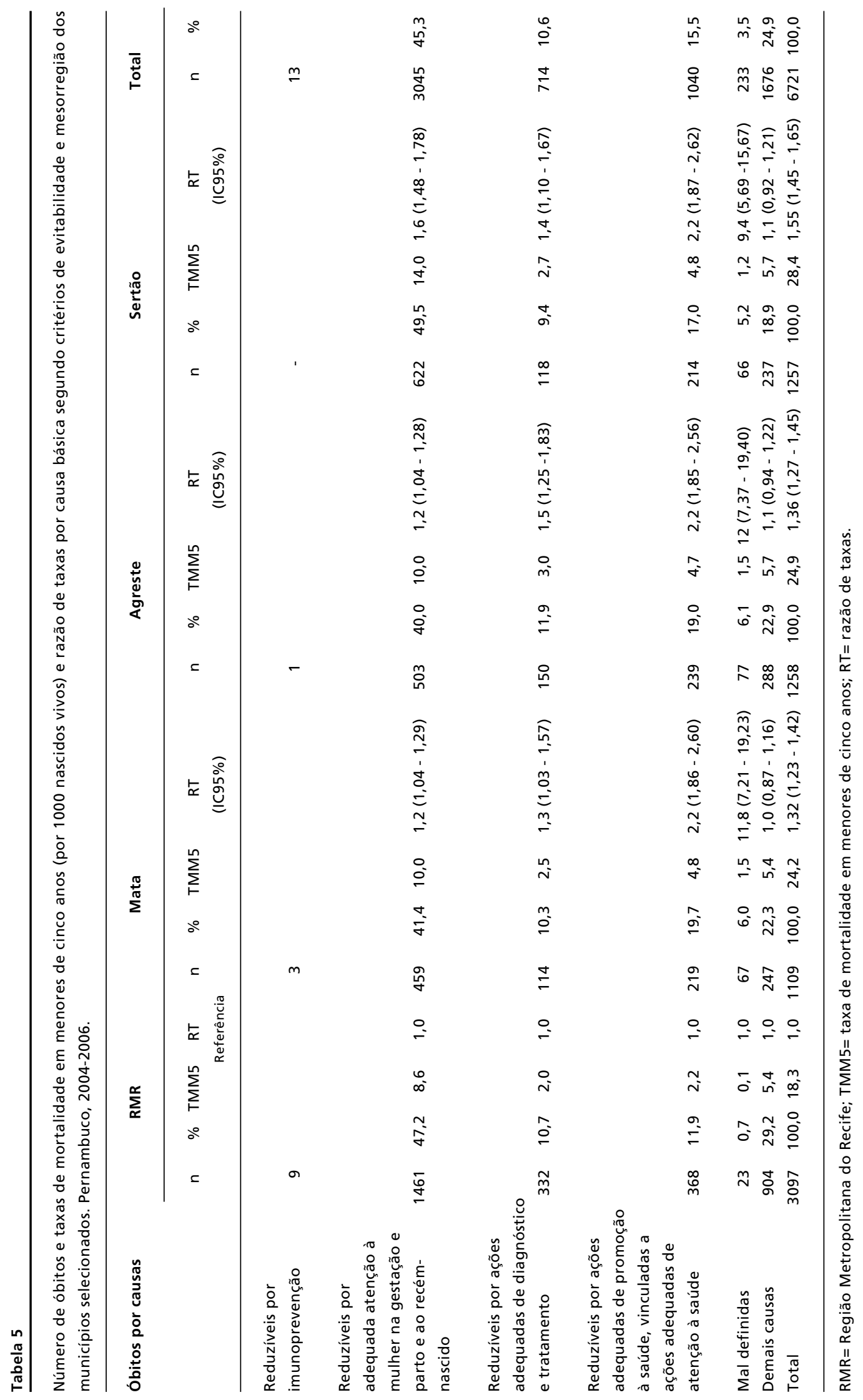


observado na RMR. No Sertão, foi encontrada a maior proporção de doenças do aparelho respiratório e de doenças infecciosas e parasitárias no grupo etário de um a quatro anos. Como causa específica, essa mesorregião apresentou cerca do dobro da proporção de acidentes de transporte observada na Mata, Agreste e RMR.

Em relação à evitabilidade das causas básicas em menores de cinco anos (Tabela 5), 71,4\% do total de mortes estudadas foram classificadas como reduzíveis por ações de saúde, com TMM5 entre 1,2 a 2,2 vezes superior na Mata, Agreste e Sertão em relação à taxa observada na RMR, dependendo da forma de redutibilidade e da mesorregião. Seriam reduzidos $45 \%$ dos óbitos caso ocorresse adequada atenção à mulher na gestação e parto e ao recémnascido. Para esse grupo de causas evitáveis, a TMM5 do Sertão foi $60 \%$ superior à observada na RMR. As causas evitáveis por ações adequadas de promoção à saúde (vinculadas a ações de atenção à saúde) apresentaram TMM5 variando entre 2,2/1000 NV (RMR) e 4,8/1000 NV (Sertão e Mata), representando $15,5 \%$ dos óbitos. As causas reduzíveis por ações adequadas de diagnóstico e tratamento foram responsáveis por $10,6 \%$ dos óbitos, com a RMR apresentando a menor TMM5 (2,0/1000 NV) e o Agreste $(3,0 / 1000 \mathrm{NV})$, a maior. Também foram identificados óbitos evitáveis por imunoprevenção, sendo nove casos na RMR, três na Mata e um no Agreste.

\section{Discussão}

A TMM5 média do conjunto dos municípios estudados sofreu influência da mesorregião mais populosa a qual contribuiu com a totalidade de seus municípios, a RMR. No entanto, essa taxa média, de $21,7 / 1000 \mathrm{NV}$, ainda se apresenta superior à TMM5 nacional (20 óbitos/1000 NV), em 2006, e muito acima das taxas em países em desenvolvimento, como Argentina (16/1000 NV), Uruguai (12/1000 NV), Chile (9/1000 NV) e Cuba (7/1000 NV), também referentes ao ano de 2006. ${ }^{14}$ Por outro lado, foi muito inferior à TMM5 estimada, referente a 2005, para o Estado de Pernambuco, de $38,6 / 1000$ NV. 15 Daí, ser provável que os 85 municípios não incluídos neste estudo, por não possuírem dados adequados para cálculo direto de taxas de mortalidade, apresentem TMM5 mais elevada do que a média dos 99 municípios estudados, o que implicaria em patamares de desigualdade superiores aos evidenciados. Ainda assim, a TMM5 das mesorregiões dos municípios selecionados apresentou-se bastante desigual, a exemplo do que ocorre entre as macrorregiões do país, nas quais a mortalidade na infância, em 2005, variou entre 16,1/1000 NV no Sul, e 38,4/1000 NV no Nordeste. 15

Apesar da TMM5 média dos municípios estudados estar próxima ao valor estipulado pela meta do milênio para 2015, existem desigualdades sociodemográficas e nas causas de morte na infância entre as mesorregiões do Estado de Pernambuco. A RMR apresentou os melhores indicadores em praticamente todas as características da mortalidade na infância analisadas, com melhor qualidade das informações sobre os óbitos, menores taxas de mortalidade e condições sócio-demográficas superiores, comportando-se, portanto, de forma particular no Estado, quando comparada às demais mesorregiões. $\mathrm{O}$ Sertão apresentou a maior taxa de mortalidade no grupo etário de menores de um ano, ao passo que, no grupo de um a quatro anos, a maior taxa de mortalidade ocorreu na Mata e no Agreste.

A proporção de cada componente etário na composição da mortalidade na infância é influenciada pela magnitude da TMM5. De acordo com Black et al.,16 populações com altas taxas de mortalidade na infância apresentam cerca de $20 \%$ de óbitos neonatais. Por outro lado, países com taxas inferiores a $35 / 1000 \mathrm{NV}$ chegam a ter mais de $50 \%$ de óbitos no período neonatal. No conjunto das mesorregiões estudadas, a mortalidade no grupo de um a quatro anos apresentou patamar muito inferior ao da mortalidade em menores de um ano. No entanto, a taxa de mortalidade na faixa de um a quatro anos apresentou-se mais desigual na Mata e no Agreste em relação ao observado em menores de um ano, sugerindo que os determinantes da mortalidade na infância atuam com diferentes intensidades nos seus dois componentes etários.

A taxa média de mortalidade em crianças de um a quatro anos apresentou-se elevada quando comparada com a situação de países em desenvolvimento, como o Chile e Cuba, com taxas de 1,6 óbitos/1000 NV, no ano de 2002.17 Ainda que a RMR tenha tido taxa de mortalidade nessa faixa etária inferior ao alcançado no país (3,00/1000 NV), em 2002,17 influenciando a taxa do conjunto das mesorregiões em direção à taxa nacional, a Mata, o Agreste e o Sertão apresentaram taxa superior à nacional.

Em relação à mortalidade infantil, a taxa estimada para Pernambuco, em 2005, foi de 32,1/1000 $\mathrm{NV}$ e para o Nordeste de $31,6 / 1000 \mathrm{NV}, 15$ valores bem acima dos encontrados neste estudo, reforçando a possível situação mais desfavorável dos 85 municípios excluídos por não disporem de dados adequados sobre nascimentos e óbitos, com índices elevados de 
sub-registro e de causas de morte mal definidas. A subenumeração dos registros vitais sugere a ocorrência de nascimentos e óbitos domiciliares e configura-se como um problema maior de ordem social, principalmente quando se traduz na invisibilidade de mortes em populações excluídas pelo poder público.

Ainda assim, a TMM5 das mesorregiões dos municípios selecionados apresentou-se bastante desigual, a exemplo do que ocorre entre as macrorregiões do país, nas quais a mortalidade na infância, em 2005, variou entre $16,1 / 1000 \mathrm{NV}$ no Sul, e $38,4 / 1000$ NV no Nordeste. ${ }^{14}$ Salienta-se que para população menor de um ano, a meta do milênio é alcançar a taxa de 15,7/1000 NV no país em 2015.15 No caso de Pernambuco, apenas a RMR apresentouse próxima dessa meta.

As diferenças na ocorrência de óbitos domiciliares entre a RMR e as demais mesorregiões sugerem deficiências e desigualdades no acesso e resolubilidade dos serviços de saúde. O óbito domiciliar deve ser considerado como uma ocorrência potencialmente evitável, tratando-se de um evento sentinela. 18

Nas mesorregiões, a desigual TMM5 entre os estratos sociodemográficos de municípios, com maiores taxas naqueles com pior situação do IDH, analfabetismo feminino e taxa de fecundidade, sugere a importância dessas variáveis na ocorrência dos óbitos na infância em Pernambuco, e reforça o impacto dos determinantes socioeconômicos sobre a mortalidade nos primeiros anos de vida.19,20 No entanto, em um mesmo estrato, as diferenças na TMM5 entre as mesorregiões, principalmente entre a RMR e as demais, sugerem também a participação de determinantes relacionados à atenção à saúde na produção desigual de óbitos na infância no Estado.

O declínio da taxa de fecundidade nas últimas décadas tem sido associado à tendência de redução da taxa de mortalidade na infância, assim como a urbanização crescente e a melhoria do nível educacional. 15 No Nordeste em 2004, a taxa de fecundidade foi de 2,39 filhos por mulher, ${ }^{21}$ situação muito mais próxima da observada na RMR do que a encontrada nas demais mesorregiões.

No Brasil, até a década de 1980 , as principais causas de morte na infância eram representadas por doenças infecciosas e parasitárias, diretamente relacionadas às condições de vida, de meio ambiente e de acesso aos serviços de saúde. Paulatinamente, as causas decorrentes de afecções perinatais, relacionadas aos transtornos da saúde materna, da gestação, do parto e do recém-nascido, passaram a ter maior participação relativa. 15 Alta proporção de óbitos por afecções perinatais expressa deficiências na assistência à gestante e ao recém-nascido, além de estar associada a precárias condições socioeconômicas. ${ }^{2}$

Muito embora, em todas as mesorregiões, as afecções perinatais tenham predominado como causa de morte no primeiro ano de vida, o Sertão, o Agreste e a Mata apresentaram proporção de óbitos por doenças infecciosas e parasitárias acima de $11 \%$, valor bem superior ao observado no país $(7,1 \%)$, em 2005.15 De outra maneira, a RMR teve como segunda causa de morte mais frequente em menores de um ano as malformações congênitas, indicando um maior controle de causas de morte evitáveis nessa mesorregião, com consequente participação mais expressiva de causas de difícil intervenção. Ainda que o Agreste também tenha apresentado como segunda causa mais frequente as malformações congênitas, esse resultado deve ser considerado com ressalvas, visto que nessa mesorregião houve elevada proporção de óbitos por causas mal definidas, o que pode ter influenciado a distribuição das outras causas.

A declaração da causa básica apresenta maior confiabilidade quando detecta o início da cadeia de eventos que levou ao óbito, fortalecendo as medidas preventivas. Entretanto, não é raro a falta de registro da doença inicial que levou ao óbito, 22 com causas intermediárias ocupando o lugar da causa básica nas declarações de óbito. Dessa forma, as afecções maternas geralmente são subestimadas, ao passo que as causas mais genéricas, como septicemia, hipóxia, baixo peso e prematuridade, são excessivamente registradas.5,22 A RMR apresentou melhor qualidade de informação sobre a causa do óbito, pois, além da menor proporção de causas mal definidas, teve simultaneamente a maior proporção de afecções maternas e a menor proporção de septicemia, hipóxia, baixo peso e prematuridade entre as mesorregiões. Contrariamente, na Mata, no Agreste e no Sertão, predominaram como causa básica "transtornos respiratórios do recém-nascido", geralmente considerada como causa intermediária. Tal situação sugere deficiências na definição e registro da causa básica nas declarações de óbito e na sua investigação pelas equipes que operacionalizam o SIM. 22

Em menores de um ano residentes na Mata, no Agreste e no Sertão, a proporção de óbitos por doenças infecciosas intestinais e por desnutrição foi o dobro da observada na RMR, indicando condições mais precárias de vida e de saneamento, além de maiores deficiências na atenção à saúde das crianças, particularmente nas ações básicas de saúde e nas 
medidas de diagnóstico e tratamento precoces, capazes de minimizar esses agravos. ${ }^{2}$

$\mathrm{Na}$ faixa etária de um a quatro anos, a preponderância das doenças do aparelho respiratório evidenciam a necessidade de melhorias de condições ambientais, como a redução de aglomeramento domiciliar, de ampliação do acesso aos serviços de saúde e de uma maior efetividade no manejo dos casos. ${ }^{5}$ As causas externas, que ocuparam o segundo lugar nesse grupo etário, em praticamente todas as mesorregiões, vem aumentando a sua importância, principalmente porque outras causas de morte tem sido melhor controladas. ${ }^{5}$ Ainda assim, as causas externas estão relacionadas às desigualdades sociais e não devem ser vistas como uma fatalidade, pois a segurança depende das condições de vida, que, por sua vez, incluem alimentação, habitação, educação, renda, meio-ambiente preservado, bem como acesso à terra e aos serviços de saúde. 23 A alta proporção de causas mal definidas na Mata dificultou a comparação entre as mesorregiões e sugere a persistência de mortes em crianças sem assistência médica. ${ }^{2}$

A maioria dos óbitos estudados poderia ser reduzida, indicando mais uma vez a necessidade de melhorias no acesso e na qualidade dos serviços de saúde. Assim, intervenções relacionadas ao aumento da cobertura e qualidade da assistência pré-natal, ao parto e ao recém-nascido, e ao manejo integrado de doenças infecciosas e respiratórias e dos problemas nutricionais apresentam grande capacidade de atuar na redução da mortalidade na infância. 5 Destaca-se ainda o elevado número de óbitos reduzíveis por imunoprevenção, sugerindo dificuldades na cobertura dos serviços de atenção básica à saúde.

O êxito na redução da magnitude e das desigualdades da mortalidade na infância, no Estado de Pernambuco, depende em grande parte da prevenção das mortes por afecções perinatais, principal causa de morte em todas as mesorregiões, sensível à redução por adequada atenção à gestação, ao nascimento e aos primeiros dias de vida da criança. Por outro lado, ainda são detectados em todas as mesorregiões óbitos considerados eventos sentinela, com grande vulnerabilidade a ações de saúde e cuja ocorrência é considerada inadmissível, como os secundários a doenças imunopreveníveis, diarréia, pneumonia e desnutrição. Os óbitos por diarréia em menores de um ano, por exemplo, além de continuarem existindo, ocorrem de forma desigual, com mortalidade proporcional nas mesorregiões do interior do Estado (Mata, Agreste e Sertão) superior à observada na RMR.

As desigualdades encontradas na mortalidade na infância, no Estado de Pernambuco, refletem o acúmulo desigual de exposições a fatores de risco e de privação a fatores de proteção pela população das diferentes mesorregiões. Coloca-se em evidência o desafio do poder público em reduzir as iniquidades, ampliando o acesso a bens e serviços de qualidade, inclusive os de saúde, com a implementação de políticas orientadas pelos princípios da universalidade e da equidade, de forma que alcancem toda a população, beneficiem aqueles que apresentam maior risco de morte e aumentem a chance de sobrevivência na infância.

\section{Referências}

1. UNICEF (Fundo das Nações Unidas para a Infância). Situação mundial da infância: saúde materna e neonatal 2009. [acesso em: 10 ago 2010] Disponível em: http://www.unicef.pt/docs/situacao_mundial_da infancia_2 009.pdf

2. Brasil. Objetivos de desenvolvimento do milênio: relatório nacional de acompanhamento. Brasília: IPEA; 2007.

3. Whitehead M. The concepts and principles of equity in health. Int J Health Serv. 1992; 22: 429-45.

4. Mosley WH, Chen LC. An analythical framework for the study of child survival in developing countries. Bull World Health Organ. 2003; 81: 141-5

5. Victora CG. Intervenções para reduzir a mortalidade infantil pré-escolar e materna no Brasil. Rev Bras Epidemiol. 2001; 4: 3-69

6. Giglio MRP, Lamounier JA, Morais Neto OL, César CC. Baixo peso ao nascer em coorte de recém-nascidos em Goiânia-Brasil no ano de 2000. Rev Bras Ginecol Obstet. 2005; 27: 130-6
7. Malta DC, Duarte EC. Causas de mortes evitáveis por ações efetivas dos serviços de saúde: uma revisão da literatura. Ciênc Saúde Coletiva. 2007; 12: 765-76.

8. Victora CG. Explaining trends in inequities: evidence from Brazilian child health studies. Lancet. 2000; 356: 1093-8.

9. IBGE (Instituto Brasileiro de Geografia e Estatística). Censo Demográfico 2000. [acesso em 10 jul 2009]. Disponível em: http://www.ibge.gov.br

10. Szwarcwald CL, Leal MC, Castilho EA, Andrade CLT, Souza Junior PRB. Estimação de mortalidade infantil no Brasil: o que dizem as informações sobre óbitos e nascimentos do Ministério da Saúde? Cad Saúde Pública. 2002; 18: $1725-36$.

11. OMS (Organização Mundial da Saúde). CID-10. São Paulo: Editora da Universidade de São Paulo; 1994

12. Malta DC, Sardinha LMV, Moura L, Lansky S, Leal MC, Szwarcwald CL, França E. Lista de causas de mortes evitáveis por intervenções do Sistema Único de Saúde do Brasil. Epidemiol Serv Saúde. 2007; 16: 231-42. 
13. Breslow NE, Day NE. Statistical methods in cancer research. Volume II: The design and analysis of cohor studies. IARC Scientific Publications 82. Lyon: International Agency for Research on Cancer; 1987.

14. UNICEF (Fundo das Nações Unidas para a Infância) Statistics by area: child survival and health: under five mortality. [acesso em 20 jul 2008]. Disponível em: http://www.childinfo.org/mortality_underfive.php

15. Brasil. Saúde Brasil 2007: uma análise da situação em saúde. Brasília: Ministério da Saúde; 2008

16. Black RE, Morris SS, Brice J. Where and why are 10 million children dying every year? Lancet. 2003; 361: 2226-34

17. OMS (Organização Mundial da Saúde). Relatório mundia da saúde 2005: para que todas as mães e crianças contem. Brasília: Organização Pan-Americana da Saúde; 2006.

18. Duchiade MP, Carvalho ML, Leal MC. As mortes em domicílio de menores de um ano na região metropolitana de Rio de Janeiro em 1986 - um "evento sentinela" na avaliação dos serviços de saúde. Cad Saúde Pública. 1989, 5: 521-63.

Recebido em 18 de março de 2010

Versão final apresentada em 21 de fevereiro de 2011

Aprovado em 12 de abril de 2011
19. Guimarães MJB, Marques NM, Melo Filho DA, Szwarcwald CL. Condições de vida e mortalidade infantil: diferenciais intra-urbanos no Recife, Pernambuco, Brasil. Cad Saúde Pública. 2003; 19: 1413-24.

20. Aquino TA, Guimarães MJB, Sarinho SW, Ferreira LOC. Fatores de risco para a mortalidade perinatal no Recife, Pernambuco, Brasil, 2003. Cad Saúde Pública. 2007; 23: 2853-61.

21. Rede Interagencial de Informação para a Saúde. Indicadores básicos para saúde no Brasil: conceitos e aplicações. Brasília: Organização Pan-Americana da Saúde; 2008

22. Mendonca EF, Goulart EMA, Machado JAD. Confiabilidade da declaração de causa básica de mortes infantis em região metropolitana do sudeste do Brasil. Rev Saúde Pública. 1994; 28: 385-91.

23. Sociedade Brasileira de Pediatria. Fórum para implantação da política nacional da redução da mortalidade por acidente e violência na Região Nordeste, 2002. [acesso em 10 jul 2009]. Disponível em: http://www.sbp.com.br/img/documentos/doc forum.pdf 